\title{
PROTEIN FOLDING
}

\section{Compartmentalizing acid stress in bacteria}

Colin Kleanthous

\begin{abstract}
A Donnan equilibrium causes an influx of chloride ions into the Escherichia coli periplasm when the bacterium finds itself in gastric fluid. The combination of low $\mathrm{pH}$ and high anion concentration drives proteins to aggregate, a potentially lethal event unless prevented by specific chaperones.
\end{abstract}

Bacteria often have to tolerate temporary discomfort en route to finding a suitable place to settle and proliferate. This is particularly the case for enteric organisms such as Escherichia coli. Following ingestion, E. coli has to withstand the highly acidic environment of the mammalian stomach on its way to the large intestine where conditions are more amenable to its commensal lifestyle ${ }^{1}$. E. coli has protein chaperones within the periplasm, a compartment separating its two membranes, which protect proteins from the acidic $\mathrm{pH}$ of gastric fluid. The work of Bardwell and colleagues in the present issue offers a surprising twist to what was thought to be a well-understood bacterial response to acid stress ${ }^{2}$. Through a series of simple yet elegant in vitro and in vivo experiments the authors show that the periplasmic chaperones are actually protecting their denatured client proteins from aggregating as a result of a surge in chloride ion concentration that accompanies acidification (Figure 1).

Bacteria are encased by one or two membranes and a rigid cell wall, which, coupled with a plethora of adaptive cellular responses, helps them stay alive when conditions become unfavorable. The periplasmic compartment of E. coli, which accounts for $\sim 35 \%$ of the cell volume, is open to the elements, in the sense that small molecules $(<600 \mathrm{Da})$ can diffuse unhindered through the narrow pores of porins that pepper the outer membrane ${ }^{3}$. As a result, 
rapid changes in environmental $\mathrm{pH}$ are quickly reflected in the periplasm. The cytoplasm by contrast is protected by its proton-impermeable membrane and several buffering mechanisms ${ }^{4}$. It is well known that aggregation of the resulting acid unfolded proteins is counteracted by periplasmic chaperones, $\mathrm{HdeA}$ and $\mathrm{HdeB}^{5}$. Hence it was surprising that none of the model periplasmic proteins and even whole periplasmic protein extracts exposed to a medium simulating gastric fluid $(10 \mathrm{mM} \mathrm{HCl} \mathrm{pH} \mathrm{2,} 150 \mathrm{mM} \mathrm{NaCl})$ aggregated. The authors rationalised that maybe the concentration of $\mathrm{Cl}^{-}$while close to that expected of gastric fluid was not reflective of that experienced by proteins within the confines of the bacterial periplasm. Indeed, raising the concentration of $\mathrm{Cl}^{-}$by just three-fold caused extensive aggregation of periplasmic proteins that was suppressed by added chaperones.

Using solute partitioning experiments to determine the fold-change in $\mathrm{Cl}^{-}$concentration relative to the external medium, Stull et al found that not only did the periplasmic volume shrink by $\sim 25 \%$ but the periplasmic $\mathrm{Cl}^{-}$concentration was elevated four-fold following acidification, well-above the levels necessary for protein aggregation at low $\mathrm{pH}$. HdeA and HdeB knockout mutants were more sensitive to chloride ions at low $\mathrm{pH}$ relative to wild-type cells and only some, but not all, periplasmic proteins were prevented from aggregating by the chaperones in vivo.

Clearly, the chaperone roles of $\mathrm{HdeA}$ and $\mathrm{HdeB}$ are more nuanced than previously realised; they are undoubtedly acid-activated but their prevention of aggregation is linked to the influx of anions to the periplasm not simply acid-induced unfolding. So what causes this surge in $\mathrm{Cl}^{-}$concentration and why does it cause aggregation? The authors suggest the Donnan effect as the likely explanation. In 1911, FG Donnan pointed out that small ions distribute asymmetrically across a semipermeable membrane if on one side of the membrane is a large, charged macromolecule that cannot move across the membrane ${ }^{6}$. In the context of the present study, the semipermeable membrane is the outer membrane of $E$. coli and the macromolecules are unfolded proteins in the periplasm that become positively charged due to acidification. The low pH protonates all carboxylate groups, breaking stabilising salt bridges 
and leaving proteins with a net positive charge due to charged histidine, lysine and arginine residues. Consistent with this interpretation, the authors found that chloride-dependent aggregation correlates with carboxylate protonation. The elevated $\mathrm{Cl}^{-}$causes aggregation by neutralizing the positive charges on the unfolded proteins, diminishing the electrostatic repulsion that would otherwise keep them apart. Furthermore, by binding to the exposed hydrophobic amino acids of these unfolded proteins, HdeA and HdeB block non-specific intermolecular associations and prevent protein aggregation (Figure 1). When the environment becomes less acidic - as E. coli cells enter a less hostile environment - the whole process reverses.

The study of Stull et al highlights how the enveloping structures of Gram-negative bacteria can influence the environmental stress they experience and raises many questions about their response to acid stress and the role of chaperones in counteracting its effects. How is the association of acid activated chaperones with client proteins influenced by the coating of chloride ions? Why are only some proteins targets for these chaperones during acid stress? Might the disruption of chaperone associations with unfolded clients offer a route towards the development of antimicrobial strategies aimed at killing Gram-negative bacteria in the acidic environment of the stomach? Are other cellular responses activated by the elevated $\mathrm{Cl}^{-}$concentration? Finally, the work opens up the possibility that there may be other instances where bacteria respond to a stress by virtue of Donnan equilibria driving the influx of ions into the periplasm.

\section{References}

1 Tenaillon, O., Skurnik, D., Picard, B. \& Denamur, E. The population genetics of commensal Escherichia coli. Nature Reviews Microbiology 8, 207-217 (2010).

2 Stull, F., Hipp, H., Stockbridge, R. B. \& Bardwell, J. C. A. In vivo chloride concentrations surge to proteotoxic levels during acid stress. Nature Chemical Biology (2018).

3 Nikaido, $\mathrm{H}$. Molecular basis of bacterial outer membrane permeability revisited. Microbiology and Molecular Biology Reviews 67, 593-656 (2003).

4 Kanjee, U. \& Houry, W. A. Mechanisms of acid resistance in Escherichia coli. Annual Reviews of Microbiology 67, 65-81 (2013). 
$5 \quad$ Zhang, S. et al. Comparative proteomics reveal distinct chaperone-client interactions in supporting bacterial acid resistance. Proceedings of the National Academy of Sciences of the United States of America 113, 10872-10877 (2016).

6 Donnan, F. G. Theorie der Membrangleichgewichte und Membranpotentiale bei Vorhandensein von nicht dialysierenden Elektrolyten. Ein Beitrag zur physikalischchemischen Physiologie. Zeitschrift für Elektrochemie und angewandte physikalische Chemie 17, 572-581 (1911).

$7 \mathrm{Yu}, \mathrm{X}$. C. et al. Characterizations of the Interactions between Escherichia coli Periplasmic Chaperone HdeA and Its Native Substrates during Acid Stress. Biochemistry 56, 5748-5757 (2017).

Figure 1. Orally-ingested, commensal Escherichia coli must first survive the acidic environment of the stomach before settling in the large intestine. Panels, The work of Stull et al shows that the low $\mathrm{pH}(\sim 2)$ of the stomach not only denatures proteins in the periplasm, causing them to become positively charged, but also results in the influx of chloride ions due to the Donnan effect (see text). The elevated $\left[\mathrm{Cl}^{-}\right]$neutralizes the charges on the proteins causing them to aggregate if acid-activated chaperones such as HdeA are not present (top panel). HdeA (and HdeB, not shown) stop aggregation of the unfolded proteins (lower pane). HdeA undergoes a dimer-to-monomer transition as part of the acid-induced activation of its chaperone activity, with multiple monomers binding a client protein?

Colin Kleanthous is in the Department of Biochemistry, University of Oxford, Oxford, UK. email: colin.kleanthous@bioch.ox.ac.uk 